\title{
Intrinsic Bias and Lineage Restriction in the Phenotype Determination of Dopamine and Neuropeptide Y Amacrine Cells
}

\author{
Sally A. Moody, ${ }^{1}$ Ida Chow, ${ }^{2}$ and Sen Huang ${ }^{1}$ \\ ${ }^{1}$ Department of Anatomy and Cell Biology, George Washington University Medical Center, Institute for Biomedical \\ Sciences, Washington, DC 20037, and 2Department of Biology, American University, Washington, DC 20016
}

\begin{abstract}
Blastomere lineages are differentially biased to produce different neurotransmitter subtypes of amacrine cells (Huang and Moody, 1995, 1997). To elucidate when this bias is acquired, we examined amacrine lineages at different early developmental times. Our experiments demonstrate that the bias to express dopamine and neuropeptide $Y$ amacrine fates involves several steps before the formation of the definitive optic cup. At cleavage stages, a retinal progenitor that contributes large numbers of cells is already biased to produce its normal repertoire of dopamine amacrine cells, as revealed by transplantation to a new location, whereas the amacrine fate of a progenitor that contributes fewer cells is modified by its new position. At neural plate stages, not all retinal progenitors are multipotent. Nearly one-half populate only the inner nuclear layer and are enriched in amacrine cells. During early optic vesicle stages, an appro-
\end{abstract}

An important issue in developmental neurobiology is how the myriad of different cell types is established. The process by which embryonic cells attain a differentiated phenotype is regulated at multiple levels and is influenced by intrinsic maternal factors as well as region-specific, tissue-specific, and cell type-specific transcription factors and cell-to-cell signaling factors (for review, see Moody, 1999a). The elucidation of how these molecules direct the fate decisions of CNS progenitors is of central importance to understanding the mechanisms by which specific phenotypes are produced.

The vertebrate retina is an important model system in which to study the cellular and molecular interactions that regulate neuronal fate-determinative events (Adler and Belecky-Adams, 1999; Perron and Harris, 1999; Reh and Levine, 1998; Cepko, 1999). Classical studies suggest that the specification of cells to become part of the retina begins via interactions between the gastrulating mesoderm and overlying ectoderm and is fixed as the eye field at neural plate stages (Saha and Grainger, 1992). However, previous events also influence retinal fate. Maternally derived factors inhibit Xenopus vegetal blastomeres from contributing to the retina, and the competence of animal blastomeres to produce the retina is regulated by their position within a field of BMP4/ Noggin signaling in the blastula (Moore and Moody, 1999).

\footnotetext{
Received Nov. 24, 1999; revised Feb. 7, 2000; accepted Feb. 10, 2000.

This work was supported by National Institutes of Health Grant EY10096. We would like to thank Ms. Lianhua Yang for all histological preparations and Kristy Kenyon and Petra Pandur for their comments.

Correspondence should be addressed to Dr. Sally A. Moody, Department of Anatomy and Cell Biology, George Washington University Medical Center, Institute for Biomedical Sciences, 2300 I Street, Northwest, Washington, DC 20037. E-mail: anasam@gwumc.edu.

Copyright (C) 2000 Society for Neuroscience $\quad 0270-6474 / 00 / 203244-10 \$ 15.00 / 0$
}

priate mitotic tree is required for dopamine and neuropeptide $\mathrm{Y}$, but not serotonin, amacrine cell clusters to form. Thus, the acquisition of amacrine fate bias involves intrinsic maternal factors at cleavage, fate restriction in the neural plate, and specified mitotic patterns in the optic vesicle. At each of these steps only a subset of the embryonic retinal progenitors contributing to amacrine subtypes is biased; the remaining progenitors maintain multipotency. Thus, from the earliest embryonic stages, progenitors of the retina are a dynamic mosaic. This is the first experimental demonstration of amacrine fate decisions that occur during early embryonic periods in advance of the events described in the later, committed retina.

Key words: serotonin; cell fate determination; neural plate; eye fields; Xenopus; retina

After embryonic cells are specified to contribute to the retina, their descendants must choose from among several phenotypes. Initial studies of the clones produced by optic cup progenitors demonstrated that they are multipotent, producing many of the different retinal cells (Holt et al., 1988; Wetts and Fraser, 1988; Turner et al., 1990). However, use of the highly stereotypic blastomeres of Xenopus cleavage embryos, in which injection of the same cell can be repeated across the experimental population to create reproducible, quantitative fate maps (Moody, 1987a,b; Huang and Moody, 1993; Moody et al., 1996), demonstrated that individual blastomeres are differentially biased to produce subsets of amacrine cells (Huang and Moody, 1995, 1997). This novel information likely was revealed because blastomeres can be consistently identified, whereas cells in the optic cup progenitor pool are heterogeneous (Cepko, 1999). Additionally, progenitor bias was revealed because the analyses focused on neurotransmitter subtypes rather than on the entire amacrine population. Amacrine cells can be subdivided into numerous subtypes based on morphology, neurotransmitter expression, and electrophysiological properties (MacNeil and Masland, 1998). If different subtypes were produced by different subsets of progenitors, analyzing the entire class would not attest to fate bias. However, investigating the clonal origin of retinal subtypes from identified progenitors established that amacrine cell fates are biased by elements of early embryonic lineages, either intrinsic or acquired via cell-cell interactions.

In this study we tested when during early development amacrine neurotransmitter fate is biased. Amacrine fate could be influenced during three important embryonic stages: maternally influenced cleavages, the specified eye field in the neural plate, and/or the committed optic vesicle. The experiments reported 
herein demonstrate that subsets of amacrine cell progenitors are biased at each stage, demonstrating the earliest steps in amacrine fate decisions.

\section{MATERIALS AND METHODS}

Egg production and selection. Fertilized Xenopus eggs were obtained by gonadotropin-induced mating of adult frogs (Moody, 1987a,b, 1999b). At the two-cell stage, embryos whose first cleavage furrow bisected the gray crescent were collected so that pigmentation could be used to identify the dorsal midline (Klein, 1987; Masho, 1990). Only those embryos with stereotypic radial cleavage patterns at the 32-cell stage (Jacobson and Hirose, 1981; Moody, 1987b) were used to ensure consistent labeling of identified progenitors (Moody et al., 1996).

Blastomere transplantation. Identified blastomeres were microinjected with $1 \mathrm{nl}$ of $0.5 \%$ Texas Red dextran amine (TRDA; Molecular Probes, Eugene, OR) at the 32-cell stage, as described previously (Huang and Moody, 1993, 1995). Unlabeled host embryos were placed in agar wells, the vitelline membranes were removed, and a single blastomere was deleted, as detailed in Moody (1999b). The labeled blastomere was dissected from the donor embryo and transplanted into the gap in the host. Embryos were cultured to tadpole stages (44-45) (Nieuwkoop and Faber, 1994).

Retinal progenitor labeling in the neural plate. The location of retinal progenitors in the eye field of the neural plate (stages 14-15) was determined according to the Eagleson and Harris (1990) fate map. Single cells were labeled by injection with intracellular microelectrodes having a resistance between 50 and $130 \mathrm{M} \Omega$. The cell resting membrane potential $(-15$ to $-70 \mathrm{mV})$ was measured to ensure that a cell was penetrated. Intracellular iontophoretic injection of 5\% TRDA in $0.2 \mathrm{M} \mathrm{KCl}$ was delivered with positive current pulses $(2 \mathrm{nA} ; 200 \mathrm{msec}$ duration; $2 \mathrm{~Hz}$ ) for $10 \mathrm{sec}$. The medial-lateral coordinates of each labeled cell were mapped by an eyepiece reticule superimposed on the neural plate fate map. The depth of the injected cell within the neural plate was recorded by use of an Inchworm Controller (Burleigh, Fishers, NY).

Blockade of mitosis. To determine whether cell division is necessary for neurotransmitter-specific amacrine clusters to form, we blocked mitosis by incubation of the embryos in a cocktail of DNA replication inhibitors, as described in detail elsewhere (Harris and Hartenstein, 1991). At stages 15, 19-21, 23/24, 25/26, 28, and 31/32, embryos were cultured in Steinberg's solution containing $20 \mathrm{~mm}$ hydroxyurea and 150 $\mu \mathrm{M}$ aphidicolin (HUA). Hydroxyurea acts within $2 \mathrm{hr}$, whereas aphidicolin requires 4-6 hr for maximal effect. The combination of the two drugs has been shown to block DNA synthesis completely in Xenopus embryos within $3 \mathrm{hr}$ of incubation (Harris and Hartenstein, 1991), which is well within the limit of one cell cycle in the retina (Jacobson, 1968; Holt et al., 1988). Embryos were maintained in this cocktail until reaching stages $45 / 46$.

Immunofluorescent detection of neurotransmitters. Tadpoles were fixed with $4 \%$ paraformaldehyde at stages $44-46$, when dopamine (DA), neuropeptide Y (NPY), and serotonin (5-HT) amacrine cells are fully discernable (Huang and Moody, 1995, 1997). Frozen sections were cut serially at $14 \mu \mathrm{m}$ and processed for immunofluorescence, as described elsewhere (Huang and Moody, 1992, 1995, 1997, 1998). Primary antibodies were against tyrosine hydroxylase (to detect DA cells; 1:400), NPY (1:200), or 5-HT (1:200; Incstar, Stillwater, MN). FITC-conjugated secondary antibodies were applied at a 1:20 dilution.

Quantitative data collection. Neurotransmitter-labeled cells were counted in every tissue section of a complete series through the eye. For those cells labeled by blastomere injection, the lineage marker and the neurotransmitter marker were simultaneously visualized with a bluegreen dual-filter set (Chromatech) at a magnification of at least $200 \times$. For neural plate-labeled clones, every tissue section was scanned as a Z-series (at $1 \mu \mathrm{m}$ steps) using a dual-laser confocal microscope (MRC1000; Bio-Rad, Hercules, CA). The entire clone was reconstructed using the Confocal Assistant software (Bio-Rad). Quantitative data were subjected to statistical tests using SigmaStat software (Jandel Scientific, Corte Madera, CA).

\section{RESULTS}

\section{The D1.2.1 blastomere amacrine lineage is intrinsically biased at cleavage stages}

Retina-producing blastomeres are differentially biased to produce specific subsets of DA, NPY, and/or 5-HT amacrine cells (Huang

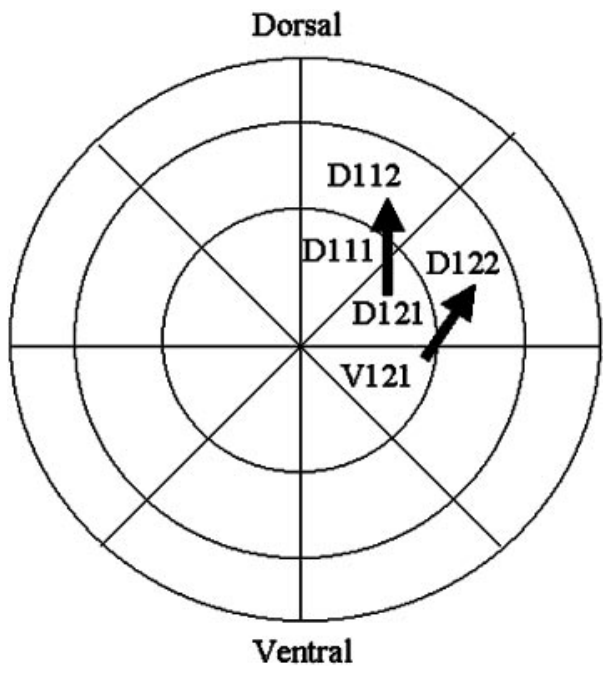

Figure 1. Diagram of the animal pole view of a 32-cell embryo. The five ipsilateral blastomeres that give rise to the retina are labeled with the Jacobson and Hirose (1981) nomenclature. Arrows demonstrate the blastomere transplantations that were performed: V1.2.1 to the position of D1.2.2 and D1.2.1 to the position of D1.1.2.

and Moody, 1995, 1997). This bias could result from the asymmetric distribution of intrinsic (i.e., maternal) factors that autonomously influence the different amacrine fates or from inductive signaling specific to the position in which the blastomere descendants differentiate. To test whether amacrine fate is intrinsically biased at cleavage stages, two blastomeres with distinct amacrine fates were transplanted to a position that normally expresses a different amacrine fate.

Blastomere V1.2.1 (Fig. 1) never produces DA amacrine cells (Huang and Moody, 1995). It was transplanted to the position of blastomere D1.2.2, which is located in an equivalent region of the BMP-signaling field (Moore and Moody, 1999) and normally produces a small number of DA cells (Huang and Moody, 1995). When V1.2.1 was transplanted to the D1.2.2 position, $60 \%$ of the embryos $(n=8)$ gave rise to DA cells, indicating that the clone took on the fate of its new position. In addition we examined the 5-HT amacrine fate of the transplanted V1.2.1 cells. Normally, D1.2.2 gives rise to significantly more 5-HT amacrine cells than does V1.2.1 (Fig. 2A) (Huang and Moody, 1997). The mean number of 5-HT amacrine cell descendants of V1.2.1 transplanted to the D1.2.2 position (Fig. $2 A, V 1.2 .1 T$ ) was statistically indistinguishable $(p>0.05)$ from that of the control blastomere of the new position $(D 1.2 .2 N)$. It was not possible to determine whether the increases in DA and 5-HT amacrine cells in the V1.2.1T lineage were at the expense of other cell types. However, the size of the transplanted blastomere's contribution to the retina (normally $61.7 \pm 14.7$ cells; $n=7$ ) significantly increased $(654 \pm 213.8 ; n=15)$ to be comparable with that of its new position $(1119.2 \pm 438.3 ; n=5 ; p<0.05)$, suggesting that other cell types would not be reduced. This experiment demonstrates that the new position of V1.2.1 allows it to express both novel DA and 5-HT amacrine cell fates and the clone size appropriate for that position. Because this blastomere changes amacrine fate when placed in a novel environment, the V1.2.1 lineage must be biased after cleavage stages. In contrast, blastomere D1.2.1 (Fig. 1) appears to be intrinsically biased at cleavage. It normally produces $32 \%$ of the DA amacrine cells in the retina (Huang and Moody, 1995). This cell was transplanted to the position of 
A. Number of LB-5HT Amacrine Cells in Clone

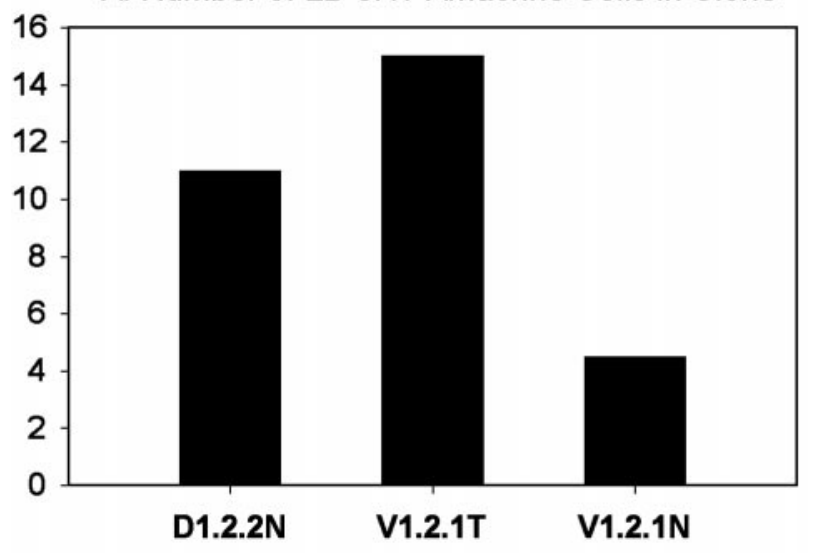

B. Number of DA Amacrine Cells in Clone

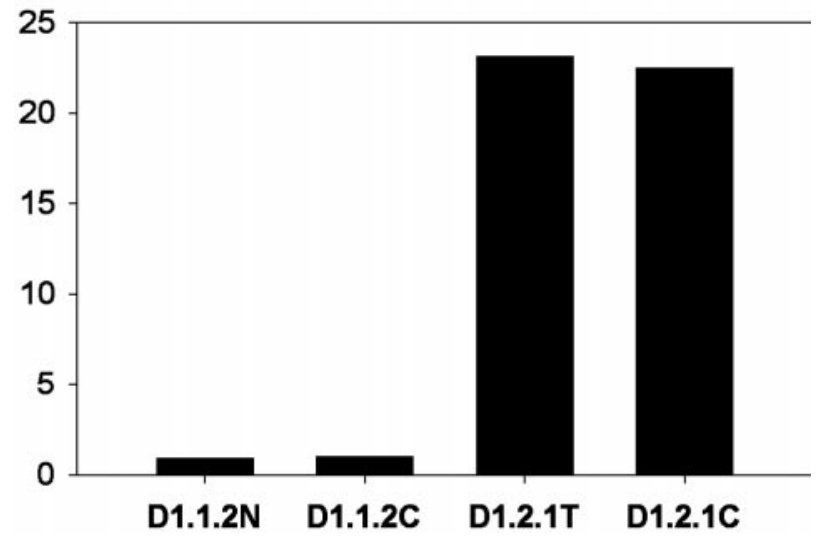

Figure 2. Changes in the neurotransmitter subtypes of amacrine cells descended from transplanted blastomeres. $A$, The number of large, bright 5-HT $(L B-5 H T)$ amacrine cells in a labeled blastomere's clone. Normally, V1.2.1 gives rise to a few $L B-5 H T$ cells $(V 1.2 .1 N)$, but after transplantation to the D1.2.2 position (V1.2.1T), it assumes a quantitative fate more similar to that of the control blastomere of its new position $(D 1.2 .2 N) . B$, The number of DA amacrine cells in a labeled blastomere's clone. Normally, D1.2.1 gives rise to $32 \%$ of the DA cells in the retina, a fate it maintains when it is control-transplanted to its normal position in the embryo (D1.2.1C). It also maintains this DA amacrine fate when it is transplanted to the D1.1.2 position $(D 1.2 .1 T)$. D1.1.2, in comparison, gives rise to a very small number of amacrine cells [normal blastomere $(D 1.1 .2 N)$; control transplanted blastomere $(D 1.1 .2 C)]$. Thus the DA amacrine fate of blastomere D1.2.1 is specified by the 32-cell stage.

blastomere D1.1.2, which is located in an equivalent region of the BMP-signaling field (Moore and Moody, 1999) and normally produced only $\sim 1 \%$ of these cells. In the D1.1.2 position, blastomere D1.2.1 produces the same number of DA amacrine cells as did D1.2.1 controls (Fig. $2 B ; p>0.05$ ). Maintenance of its original DA amacrine fate in a new environment indicates that the D1.2.1 lineage is intrinsically biased at cleavage stages, a period of development that relies entirely on maternal transcripts and proteins (Newport and Kirschner, 1982). These experiments demonstrate that the blastomere progenitors of the retina are a mosaic of intrinsically biased (D1.2.1) and positionally biased (V1.2.1) cells with regard to the production of neurotransmitter subsets of amacrine cells.

\section{Some neural plate progenitors produce clones} restricted to the inner nuclear layer

Those blastomeres (e.g., V1.2.1) that are not biased to their characteristic amacrine fate during cleavage stages must acquire bias later in development. The eye field, that region of the neural plate committed to give rise to the retinas, is specified by interactions occurring during gastrulation (Adelmann, 1937; Spemann, 1938; Saha and Grainger, 1992). Therefore, the neural plate stage is the next likely time at which the amacrine fate bias of blastomere lineages may occur. The eye field can be accurately located (Eagleson and Harris, 1990), and single cells within it can be injected with lineage tracer. Out of 109 neural plate cells labeled, only 5 clones contained cells outside the retina. To ensure that the labeled clones were derived from a single progenitor, we fixed some embryos immediately after dye injection. In every case $(n=10)$ a single progenitor was labeled (Fig. $3 A)$. Because the molecular weight of the lineage dye is too large to pass through gap junctions, labeled cells identified in the tadpole retina represent the progeny of a single neural plate progenitor cell.

Approximately one-half (56.7\%) of the clones generated from eye field progenitors and identified in the tadpole retina (total $=$ 94) were arranged in radial columns across the layers of the retina (Fig. 3B), similar to clones derived from progenitors in the optic cup (Holt et al., 1988; Wetts and Fraser, 1988). Most of these clones were arranged into tight columns, but $3.3 \%$ also contained a few dispersed cells, as depicted in Figure $3 B$; this is comparable with observations in chick and mouse (Fekete et al., 1994; Reese and Tan, 1998). In contrast to these studies, however, a large number of the clones (43.3\%) were arrayed laterally, approximating the retinal layers (Fig. $3 C, D$ ). To quantify the layer-specific distribution of the neural plate clones, we divided the retina into four layers: the ganglion cell (GC) layer, the amacrine cell (AM) layer [inner sublamina of the inner nuclear layer (INL)] (Huang and Moody, 1998), the bipolar/horizontal/Müller cell (BH) layer (outer sublamina of the INL), and the photoreceptor (PH) layer. Each clone was classified as (1) confined to a single layer, (2) confined to two adjacent layers, (3) distributed to two nonadjacent layers, (4) distributed to three layers, or (5) distributed to four layers (Fig. 3D). We refer to the first two categories as "layered" clones and to the last three categories as "radial" clones, which constitute the radial columns mentioned above. Some radial clones spanned all four layers, but the majority spanned three layers; only a small number of clones were distributed to two nonadjacent layers (Fig. 4A). Approximately one-half of the layered clones $(42.6 \%)$ populated only one layer, and approximately one-half $(53.6 \%)$ consisted of cells in adjacent layers (Figs. 3C,D, 4). The majority of single-layer clones occupied the $\mathrm{BH}$ layer, and the majority of adjacent layer clones were restricted to the INL (AM plus BH) (Fig. 4B). These data indicate that at neural plate stages there are a significant number of novel progenitors that are partially restricted in fate, particularly to cells of the INL.

Smaller, restricted clones could result from technical artifacts. Intracellular injection of a small neuroepithelial cell could damage it, causing selective cell death. To ensure against this, clones were periodically viewed with low-light epifluorescence, and those showing signs of damage were eliminated from the sample. Intracellular labels can become diluted over a large number of cell divisions so that the later products of the lineage are not visualized. This is not likely to affect the data reported herein because numerous studies have shown that labeled dextrans persist for at least $7 \mathrm{~d}$ of development in Xenopus. This encompasses at least 14 cell divisions and $3 \mathrm{~d}$ of development beyond the developmental period of the neural plate clones studied in this report. Furthermore, all data were collected by the highly sensitive technique of 


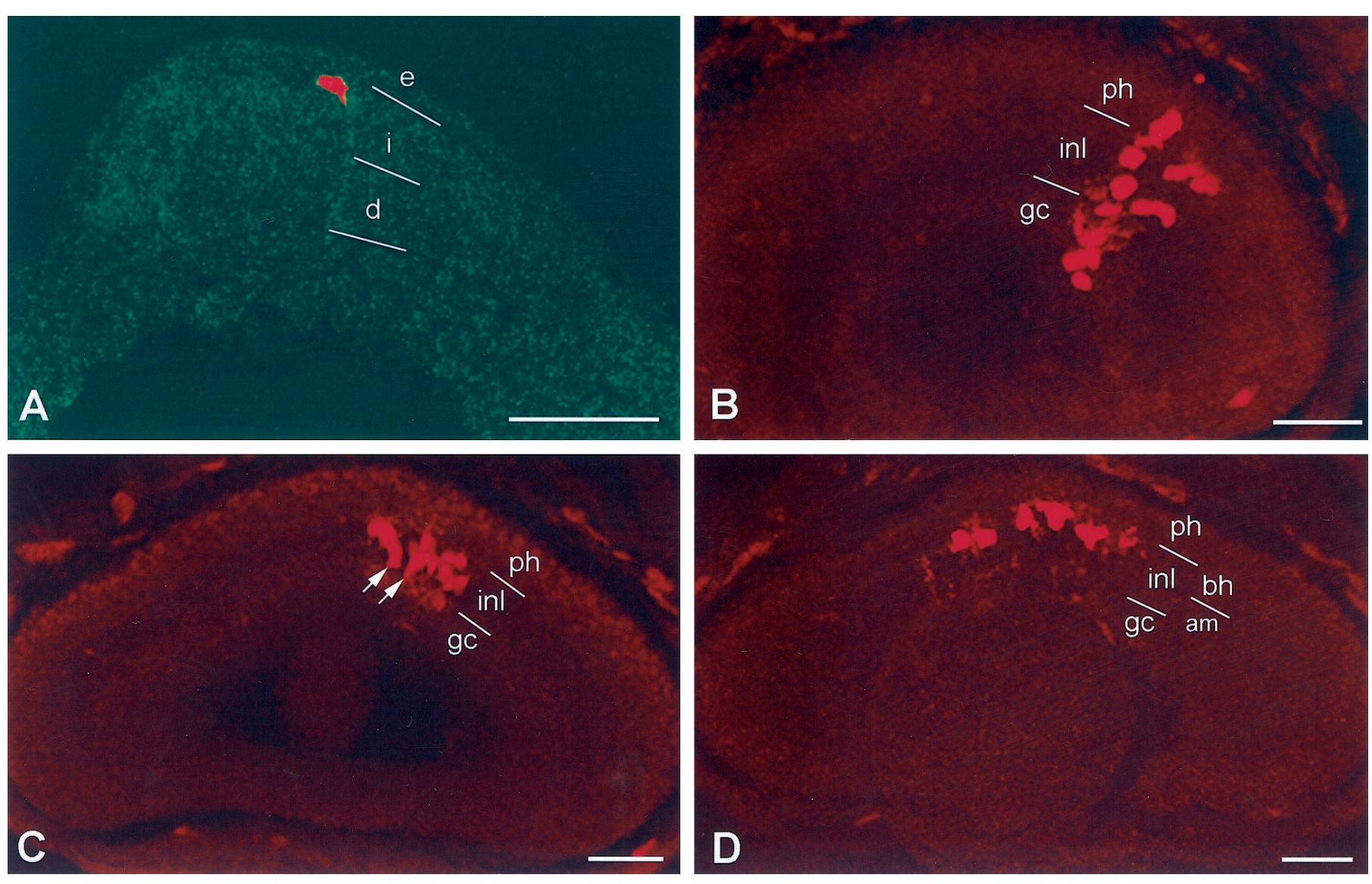

Figure 3. Clones resulting from the intracellular labeling of single cells at neural plate stages. $A$, An embryo fixed 10 min after labeling. Only a single cell was injected with tracer $(r e d)$. This sagittal section demonstrates that in the region of the eye field, the neural plate is approximately four to six cells thick and can be divided into an intermediate zone $(i)$ and a deep zone $(d)$. The superficial zone is comprised of non-neural ectoderm $(e)$. $B$, A section through the stage 44 retina demonstrating a radial clone (red column) containing cells in each retinal layer. This clone also contains a few dispersed cells, a configuration only observed in 3.3\% of cases and consistent with reports in chick and mouse (Fekete et al., 1994; Reese and Tan, 1998). C, A layered clone that resides entirely in the INL. It contains cells in the outer sublamina and amacrine cells (arrows) in the inner sublamina. $D$, A layered clone that resides entirely in the outer sublamina of the INL. $a m$, Inner sublamina of the INL; $b h$, outer sublamina of the INL; $g c$, ganglion cell layer; inl, inner nuclear layer; $p h$, photoreceptor layer. Scale bars, $100 \mu \mathrm{m}$.

averaged confocal microscopy, so that lightly labeled cells would be identified. We did not observe clones containing cells of mixed fluorescent intensities, which would indicate differential loss of label or passage of dye between unrelated cells, or fluorescent debris, which would indicate cell loss. Finally, because of the spherical shape of the retina, it is possible to misidentify the layer distribution of cells at areas of high curvature, especially near the periphery. However, because these clones were initiated early they all occupied the central retina, which in the tadpole is clearly laminated, rendering assignment to cellular layers very accurate. Furthermore, clones were reconstructed in three dimensions from serial sections to ensure accuracy of laminar identity. Therefore, it is unlikely that technical artifacts account for the observed layered clones.

The labeled neural plate cell from which each clone descended was assigned coordinates based on its medial versus lateral position at the time of injection to determine whether radial or layered progenitors occupy different regions of the eye field. Both the lateral and medial parts of the eye field produced layered and radial clones in a statistically indistinguishable pattern (Fig. 5; $p>0.1$ ). Because the sensorial (inner) layer of the neural plate, from which the neuronal elements arise, is $\sim 80 \mu \mathrm{m}$ thick (or four to six cell diameters) in the region of the eye field, it was divided into intermediate ( $<40 \mu \mathrm{m}$ from the surface) and deep $(>40 \mu \mathrm{m})$ sublayers (Fig. $3 A$ ). Progenitors of both layered and radial clones were distributed throughout the depth of the eye field, although radial clone progenitors were found slightly more often at an intermediate depth (Fig. 5). These data demonstrate that location within the eye field does not induce either of these progenitor types. They, however, do not exclude that a local inductive signal could have occurred previously, before the dispersion of the induced cells. It has been demonstrated, for example, that blastomere clones remain coherent until late blastula and then disperse and intermingle (Jacobson and Hirose, 1978; Wetts and Fraser, 1989; Bauer et al., 1994). This analysis further shows that the two types of eye field progenitors are spatially heterogeneous.

The sizes of the labeled clones were determined at tadpole stages (44/45) when neurogenesis is nearly complete; $95 \%$ of retinal cells are generated by stage 38 (Holt et al., 1988). Overall, neural plate clones are twice the size of optic cup clones (mean $=$ 8 vs $<4$ cells) (Holt et al., 1988), indicating that retinal progenitors undergo approximately one to two cell divisions during the $20 \mathrm{hr}$ interval between neural plate and optic cup stages. This is consistent with the 6-12 hr cell cycle time estimated by birthdating studies (Jacobson, 1968; Holt et al., 1988). Comparison of the size of layered versus radial clones, however, indicated that 


\section{A. Radial Clone Distribution}

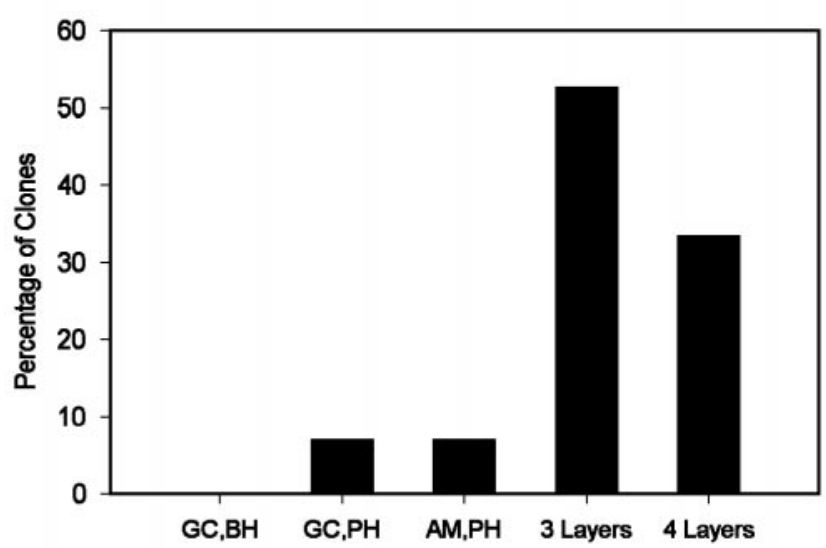

B. Layered Clone Distribution

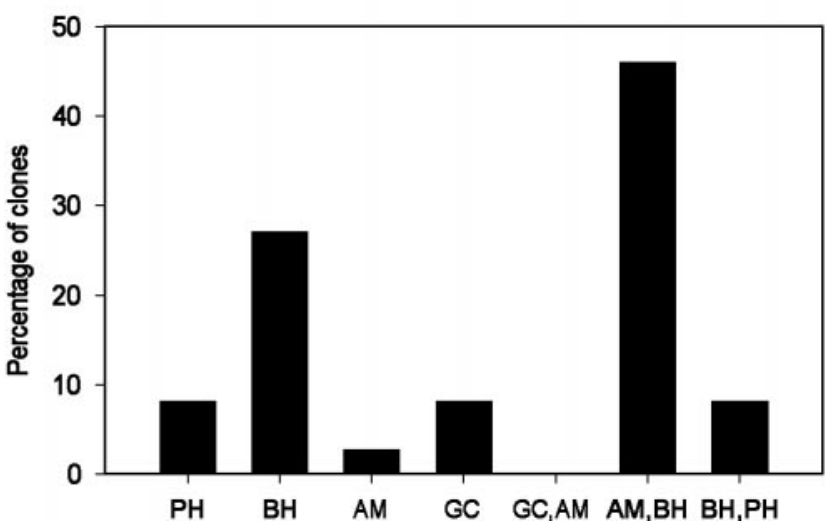

Figure 4. The distribution of cells in clones descended from neural plate progenitors. $A$, The percentage of radial clones containing progeny in two nonadjacent layers (GC,BH; GC,PH; AM,PH), in three layers, or in four layers. $B$, The percentage of layered clones containing progeny in single layers (left side) or two adjacent layers (right side). In both categories, layered clones are found predominantly in the inner nuclear layer $(\mathrm{BH}$; $\mathrm{AM} ; \mathrm{AM}, \mathrm{BH})$.

radial clone progenitors go through more rounds of cell division between neural plate and tadpole stages than do layered clones. Each clone was categorized as being produced from a minimum of one cell division ( 2 cells in clone), two cell divisions (3-4 cells), three cell divisions (5-8 cells), four cell divisions (9-16 cells), or five cell divisions (17-32 cells), assuming that cell divisions are symmetric and cell death is minimal [as per Holt et al. (1988)]. Clones confined to a single layer are smallest in size $($ mean $=2.7)$, and most $(88 \%)$ divided only one or two more times after labeling (Fig. 6). Those few that divided more than twice were found only in the $\mathrm{BH}$ layer, in which the largest number of cell classes exists. Clones confined to two adjacent layers were larger (mean $=8.0)$ and divided an average of three more times. Radial clones were slightly larger yet (mean $=9.1$ ). All but $8 \%$ divided a minimum of three times, and nearly onehalf divided four or more times (Fig. 6). These data demonstrate that the eye field progenitors are a temporal mosaic; i.e., they are a mixture of cells with different numbers of cell divisions remaining before their terminal mitosis. Furthermore, there is a correlation between the number of cell divisions remaining and the number of layers in which the cells in the clone reside. More restricted (i.e., layered) clones of the neural plate are closer to their terminal mitoses.

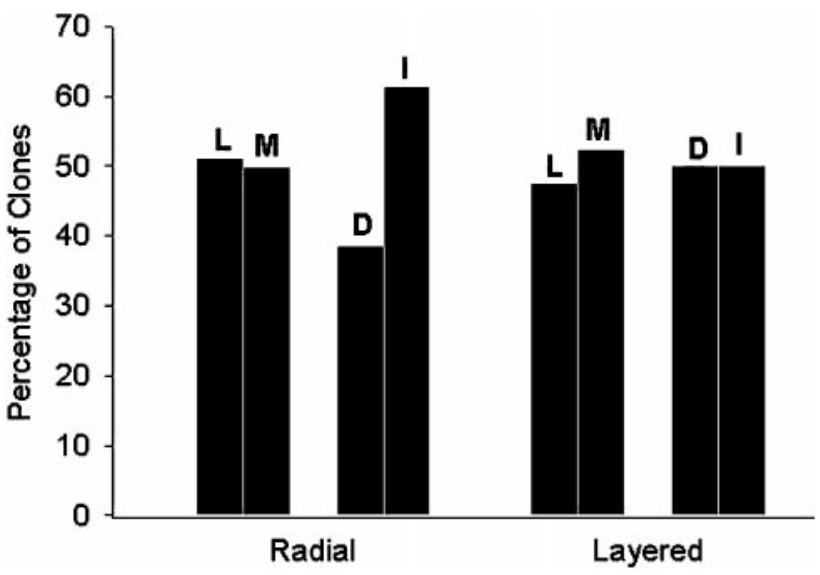

Figure 5. The spatial distribution of eye field progenitors that give rise to radial and layered clones. Both radial and layered progenitors are equally distributed between lateral $(L)$ and medial $(M)$ regions of the eye field. Radial progenitors are found slightly more frequently in intermediate $(I$, $<40 \mu \mathrm{m})$ versus deep $(D,>40 \mu \mathrm{m})$ cell layers, whereas layered progenitors are equally distributed throughout the depth of the neural plate.

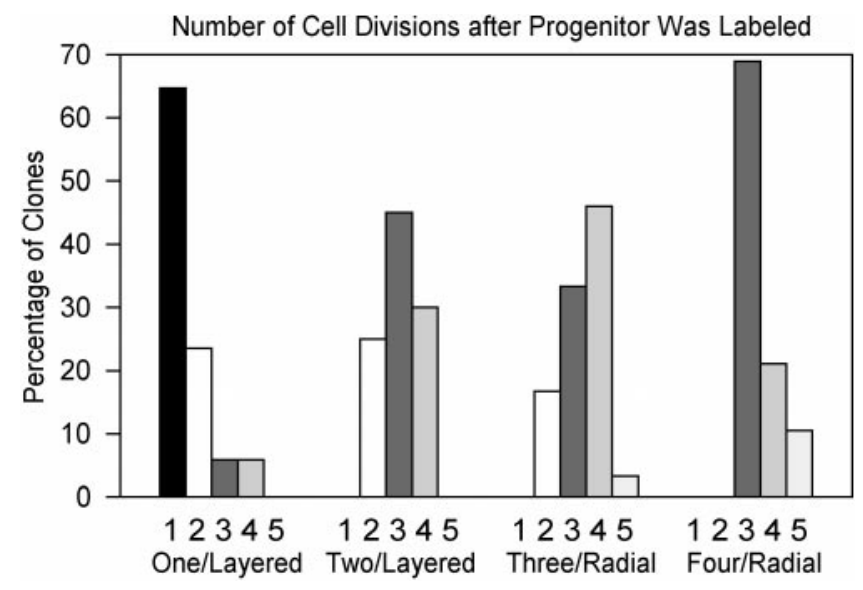

Figure 6. Layered clone progenitors divide fewer times than do radial clone progenitors. The number of cell divisions that occurred after a neural plate progenitor was labeled was determined by the size of the clone. The percentage of clones in each size bin (1-5 cell divisions) is shown for layered clones confined to one layer (One/Layered), layered clones confined to two adjacent layers (Two/Layered), radial clones distributed to three layers (Three/Radial), and radial clones distributed to four layers (Four/Radial).

\section{Clusters of neurotransmitter subtypes of amacrine cells are lineally related}

Previous studies suggested that lineage-restricted factors might influence DA, NPY, and 5-HT amacrine cell fate choices. Each subtype descends from a unique subset of blastomeres, and each differentiates in small clusters that may be clonally related (Huang and Moody, 1995, 1997). These amacrine subtypes first differentiate as single cells, scattered across the INL (Huang and Moody, 1995, 1997). As more cells differentiate, they form small clusters. These clusters may result from local inductive cues from neighboring cells. Alternatively, an eye field progenitor may be intrinsically biased to produce a specific subtype of amacrine cell, based on its mitotic pattern. We first assessed whether clones enriched in amacrine cells, i.e., those that potentially could give rise to these clusters, preferentially descend from layered versus radial eye field progenitors. Nearly one-half of the clones con- 


\section{A. Percentage of Clones Containing More Than One Amacrine Cell}

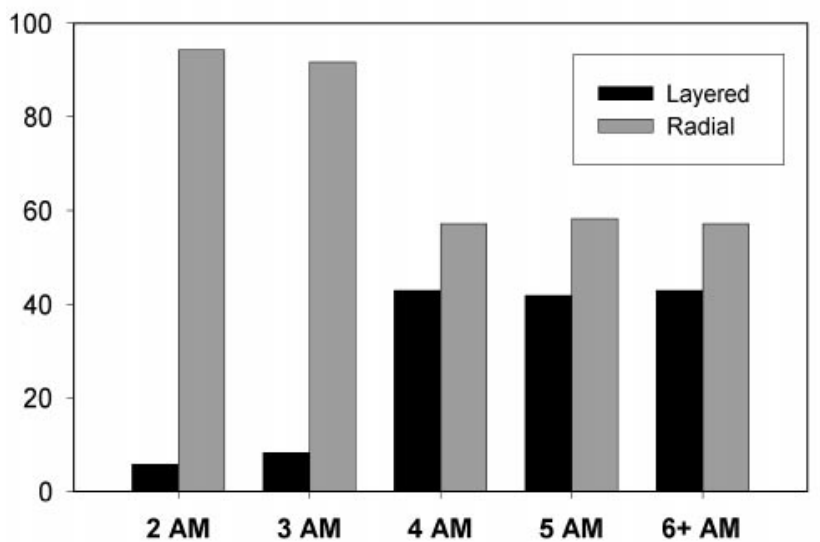

B. Percentage of Siblings to Amacrine Cells In Layered Clones

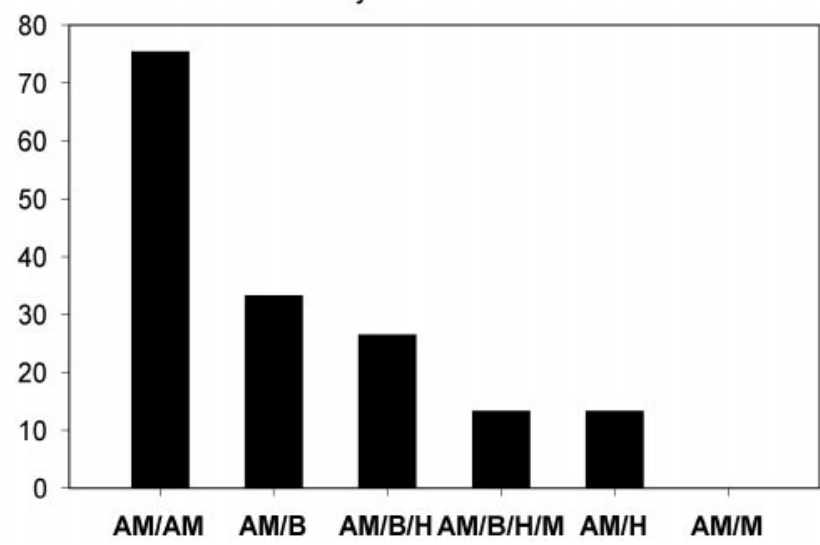

Figure 7. Layered clones are enriched in amacrine cells. A, Clones derived from eye field progenitors that contained more than one amacrine cell $(A M)$ were analyzed as either radial (gray bars) or layered (black bars). The majority of clones containing small numbers of amacrine cells were radial, whereas nearly one-half of the clones containing large numbers of amacrine cells were layered. $B$, In layered clones, amacrine cells most frequently were siblings of other amacrine cells. They also were commonly siblings of bipolar cells $(B)$, either alone or in common with horizontal $(H)$ and Müller $(M)$ cells. Rarely were amacrine cells only siblings of horizontal cells, and never were they siblings of only Müller cells.

taining four or more amacrine cells arose from layered clones (Fig. 7A). Because these clones averaged less than eight cells, they were composed of a minimum of one-half amacrine cells. In contrast, clones containing less than four amacrine cells preferentially descended from radial clones. Furthermore, amacrine cells most frequently were siblings of other amacrine cells (Fig. $7 B$ ), although only one clone contained only amacrine cells. The enrichment of amacrine cell membership in layered clones supports the possibility that a subset of eye field progenitors gives rise to the previously described DA, NPY, and/or 5-HT amacrine cell clusters.

These data are consistent with the hypothesis that neurotransmitter-specific clusters of amacrine cells arise within a layered clone lineage. To test this directly, one should evaluate the neurotransmitter expression of amacrine cells in clones derived from eye field progenitors. However, because these phenotypes are rare (each comprises $<1 \%$ of the total retinal popula- tion) (Huang and Moody, 1995, 1997) and it is not possible to target sampling to the layered clone progenitors (Fig. 5), we were unable to obtain these data. Therefore, an alternative strategy was used to address whether amacrine neurotransmitter clusters are lineally related. To identify whether a mitotic tree is required, we blocked cell division by treatment with a cocktail of DNA replication inhibitors (HUA) (Harris and Hartenstein, 1991). Clusters should still form if they result from local inductions, whereas they should be repressed if they are specified by a lineal mechanism. Blocking mitosis beginning at neural plate stages (stage 15) significantly suppressed the formation of the retina, as described previously (Harris and Hartenstein, 1991). In contrast, beginning HUA treatment when the first morphological sign of the optic vesicle is evident (stages 19-21) allowed a small retina to form. Over one-half (12 of 21) of these contained no DA amacrine cells. The rest contained only scattered, single DA amacrine cells (i.e., no clusters), and the mean number of DA amacrine cells per retina was significantly reduced $(1.2 \pm 0.3)$ from normal $(54.6 \pm 2.1 ; p<0.01)$. These data indicate that the first amacrine cells of a future cluster are produced during the initial formation of the optic vesicle. To test for local inductive versus lineage influences on amacrine neurotransmitter cluster formation, therefore, we analyzed embryos treated with HUA at subsequent stages so that only mitoses after this initial period of amacrine production were blocked.

Blocking mitosis beginning in the intermediate optic vesicle (stages 23/24) virtually eliminated the proportion of DA and NPY amacrine cells found in clusters (Fig. 8A,B). No DA clusters were found in 8 of 10 embryos. One embryo contained one two-cell DA cluster, and one embryo contained two two-cell DA clusters, compared with controls in which 14 of 14 embryos contained an average of $11( \pm 0.5)$ DA clusters. No NPY clusters were found in 9 of 12 embryos, and in the remaining 3 embryos each contained one two-cell cluster, compared with controls in which 12 of 12 embryos contained an average of $4.8( \pm 0.4)$ NPY clusters. When HUA treatment was begun in the late optic vesicle (stages 25/26), the percentage of cells found in clusters remained significantly lower than normal (Fig. 8A,B). One-half of the embryos (5 of 10) contained an average of two DA clusters, and 3 of 10 contained one NPY cluster. When HUA treatment was begun at the beginning of vesicle invagination (stage 28), approximately one-third of the normal proportion of DA cells and $\sim 44 \%$ of the normal proportion of NPY cells were found in clusters (Fig. $8 A, B$ ). The majority of embryos contained at least one two-cell cluster of DA ( $n=9$ of 10; mean number of clusters per embryo $=2.2 \pm 0.3$ ) or NPY (7 of 12 ; mean number of clusters per embryo $=0.9 \pm 0.7)$ amacrine cells. This large reduction in the number of clusters per retina compared with normal $(p<0.05)$ indicates a significant repression of cluster formation. When HUA treatment was begun in the definitive optic cup (stages 31/32), $90 \%$ of embryos (9 of 10) contained DA amacrine clusters that constituted $50.3 \%$ of the normal proportion of DA amacrine cells in clusters (Fig. $8 A$ ). A majority of embryos (7 of 10) contained NPY clusters that constituted $36.2 \%$ of the normal proportion of NPY cells in clusters. Thus, the formation of clusters is less affected when blockade is delayed until early optic cup stages. However, the number of clusters per retina still was reduced significantly $(2.9 \pm 1.7$ for DA and $1.2 \pm$ 0.8 for NPY; $p<0.05$ ), which is not surprising because the Xenopus embryonic retina continues to divide through stage 38 (Holt et al., 1988). These data demonstrate that the initial for- 
A. Percentage of DA Cells in Clusters

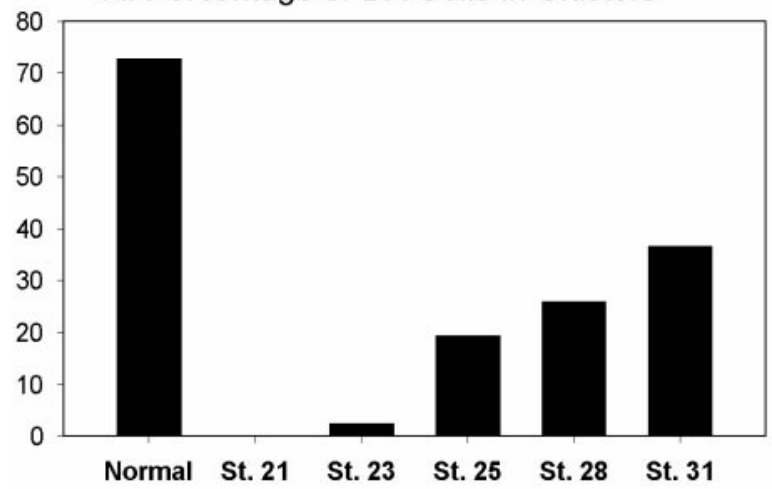

B. Percentage of NPY Cells in Clusters

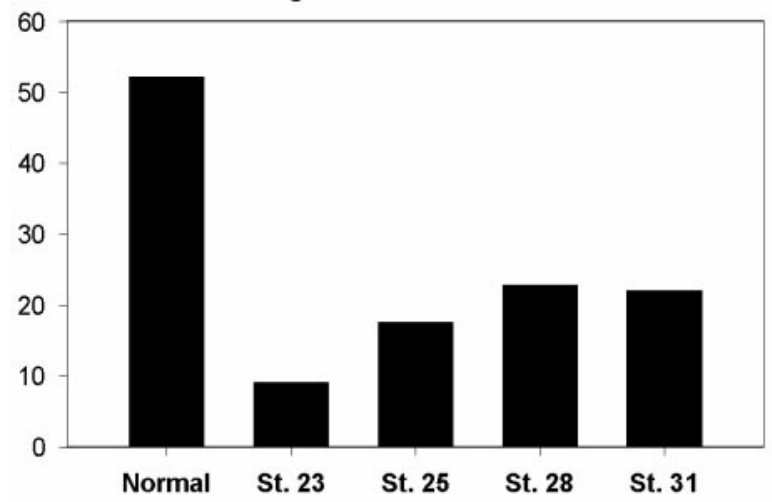

C. Percentage of $5 \mathrm{HT}$ Cells in Clusters

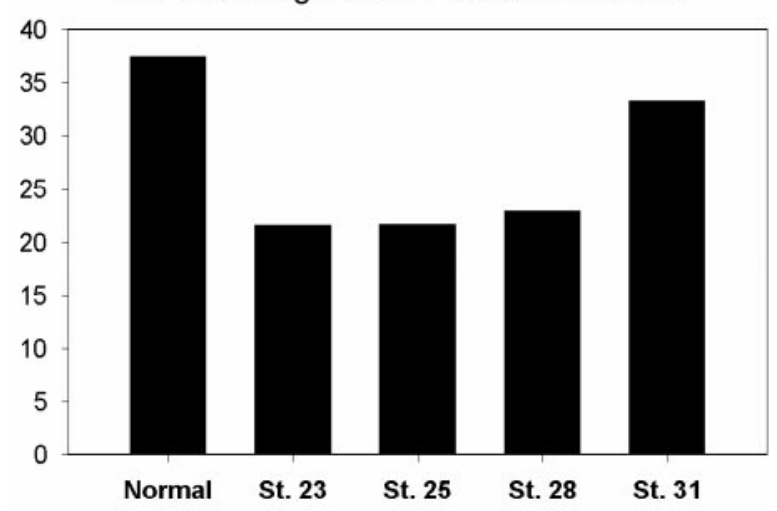

Figure 8. Formation of DA and NPY amacrine clusters is inhibited when mitosis is repressed during optic vesicle stages. $A$, The percentage of DA amacrine cells found in clusters is completely inhibited when embryos are incubated in DNA replication inhibitors starting at stage 21 . The percentage of clusters remains significantly repressed during optic vesicle stages (23-25) and gradually returns to nearly $50 \%$ of the normal level by optic cup stages (31). $B$, The percentage of NPY amacrine cells found in clusters is significantly repressed during optic vesicle stages (23-25) and returns to nearly $50 \%$ of the normal level by optic cup stages (31). $C$, The percentage of LB-5-HT amacrine cells found in clusters is reduced to slightly $>50 \%$ during optic vesicle stages and returns to near normal by optic cup stages. St., Stage.

mation of DA and NPY amacrine cell clusters depends on cell division initiated in the optic vesicle between stages 23 and 28 .

Large, bright 5-HT (LB-5-HT) amacrine cells also develop in clusters (Huang and Moody, 1997), but in contrast to DA and
NPY clusters, LB-5-HT clusters were not completely eliminated by cell division blockade at optic vesicle stages (Fig. $8 C$ ). When HUA treatment was begun at stage 23/24, the proportion of cells found in clusters was reduced to $57.3 \%$ of the normal level. This proportion increased to $89 \%$ of the normal level when treatment was begun at optic cup stages. Because simply blocking the terminal cell division could produce this level of reduction, these data do not strongly support a lineage mechanism for LB-5-HT cluster formation. Thus, the mechanisms regulating the production of the different neurotransmitter subtypes of amacrine cells vary from one subtype to another. Because 5-HT fate also changed after V1.2.1 transplantation, the 5-HT phenotype in general may depend on cellular interactions.

\section{DISCUSSION}

The attainment of a differentiated phenotype occurs via a number of progressively restrictive fate decisions made by a combination of intrinsically determined mechanisms and cell-cell interactions. Although many studies describe important interactions that affect retinal fate during optic cup and later stages (Adler and Belecky-Adams, 1999; Perron and Harris, 1999; Reh and Levine, 1998; Cepko, 1999), little is known about fate decisions in the retinal pathway before optic cup stages. Previous analyses of blastomere contributions to DA, NPY, and 5-HT amacrine cells demonstrated that retina-producing blastomeres are differentially biased to produce subsets of these neurotransmitter subtypes (Huang and Moody, 1995, 1997) but did not establish the developmental time at which this occurs. This bias could result from maternal factors acting at cleavage stages and/or from interactions at later stages.

\section{Maternal factors influence the earliest steps in embryonic retinal lineages}

Because those specific blastomeres that produce the retina in Xenopus embryos can be identified, it is possible to test their fate commitment by deletion and transplantation experiments. The extent of a blastomere's contribution to the retina depends on both its position within the field of neural inductive signaling and localized maternal factors that inhibit vegetal blastomeres from contributing to the retina (Huang and Moody, 1993; Moore and Moody, 1999). In this study we provide further evidence of a maternal influence on retinal fate. D1.2.1 expressed its normal, large number of DA amacrine cells after it was transplanted to the position of an equatorial blastomere that normally produces few of these cells. The maintenance of its DA amacrine fate after a manipulation done several hours before the onset of zygotic transcription reveals an intrinsic bias and early amacrine fate specification within the D1.2.1 lineage. However, not all blastomeres that are biased to produce specific subsets of amacrine cells are influenced by maternal factors (e.g., V1.2.1), illustrating that the retina-producing blastomeres are a mosaic of intrinsically biased and positionally specified progenitors.

\section{Some eye field progenitors are biased to produce INL cells}

Because not every retina-producing blastomere is maternally specified to produce its specific subsets of amacrine cells, when do these cells become biased? Because classical studies suggest that the specification to become retina occurs from the gastrula to the neural plate, the eye field progenitors were a likely population in which amacrine fate bias would be detected. In fact, nearly onehalf of eye field progenitors produced clones that are restricted to the INL. And many of the INL clones contained an enriched 
proportion of amacrine cells. Furthermore, amacrine cells in INL clones most frequently are siblings of other amacrine cells. This distribution pattern is distinctly different from that of radial clones in which no enrichment for amacrine cells was detected (this study) (Wetts and Fraser, 1988; Wetts et al., 1989), suggesting that some progenitors (layered) acquire an amacrine bias via the interactions that establish the eye field.

It is interesting that a significant number of neural plate cells produce layer-restricted clones, whereas progenitor cells in later retinal structures are reported to be mostly multipotent. Because clones established at the beginning of development also are primarily large, radially oriented blocks of cells (Williams and Goldowitz, 1992a; Huang and Moody, 1993), it has been assumed that the radial, multipotent clone is the building block of the retina. If this were true, progenitors labeled at progressively later time points would all be radial but progressively smaller. For the most part this fits the published data from optic cup and later stages (but see Williams and Goldowitz, 1992b). But, by labeling cells before the optic cup stage, we demonstrate a discontinuity between early radial blocks and later radial columns. Clones derived from early chick optic vesicle also are not strictly radial (Fekete et al., 1994), having single cells dispersed among multiple radial columns. It is not clear whether these dispersed cells migrated from the radial columns (Reese and Tan, 1998) or represent the last divisions of layered progenitors. Our data do not support the idea that the cells comprising a layered clone are derived from a radial column, because very few radial columns were associated with dispersed cells. This lack of intermediate clones implies instead that there is a mechanism that instructs some eye field progenitors to express a limited repertoire, and their frequency suggests that this selection between layered and radial may be random. Because layered clones virtually disappear by optic cup stages, either the layered lineages mostly have reached their mitotic termination by then (as indicated by having fewer mitoses), or they inhibit neighboring progenitors from expressing the same restricted fate (e.g. Waid and McLoon, 1998; Belliveau and Cepko, 1999).

\section{The pool of eye field progenitors is mosaic}

Although previous lineage studies indicated that most progenitor cells in the optic vesicle and cup produce nearly all retinal cell types, recent in vitro studies suggest that the progenitor pool contains differentially biased precursors (Alexiades and Cepko, 1997; Jensen and Raff, 1997; Marrow et al., 1998; Belliveau and Cepko, 1999). In fact, the concept that retinal lineages are not homogeneously multipotent but are differentially biased was elegantly demonstrated by a statistical analysis (Williams and Goldowitz, 1992b) of published data (Turner et al., 1990); the frequency of clones containing only two cell types was much higher than expected, and that of clones extending across all layers was much lower than expected. Recently, a model has been put forward to reconcile the substantial documentation of extrinsic influences on retinal fate choices and the evidence of fate-biased progenitors (Cepko, 1999). It proposes that retinal progenitors pass through a series of determinative states, each of which is intrinsically specified to respond to particular environmental cues that influence the cell types produced. For example, embryonic retinal progenitor cells differ in many characteristics from neonatal ones (Watanabe and Raff, 1990; Lillien and Cepko, 1992; Waid and McLoon, 1995; Alexiades and Cepko, 1997; Marrow et al., 1998), and these states can be influenced by the presence of other cell populations (Reh and Tully, 1986; Reh, 1992; Austin et al., 1995; Waid and McLoon, 1998; Belliveau and Cepko, 1999) and cytokines (Harris, 1997).

Our observations, obtained from normally developing embryos, are consistent with the cultured cell model, extend its application to earlier points in the retinal lineage (cleavage and neural plate), and validate its application to the intact embryo. There are at least two kinds of eye field progenitors, those that produce radial clones and those that produce layered clones. These two progenitors differ in the layer distribution of their constituents, their cellular complexity, and the number of cell divisions remaining in their respective lineages. Thus, radial and layered eye field progenitors likely represent two different determinative states. Alternatively, they represent separate lineages for the early-formed primary (layered) versus later-formed secondary (radial) retina, similar to what has been described for neural plate progenitors of primary and secondary spinal neurons (Hartenstein, 1989).

\section{DA and NPY amacrine cells form clusters via a lineage mechanism acting in the optic vesicle}

It was not technically feasible to demonstrate that layered clone progenitors produce only one neurotransmitter subtype of amacrine cell. Instead, we used the observations that at the initial stages of neurotransmitter expression in the retina, DA, NPY, and 5-HT amacrine cells are scattered as single cells across the INL and then are joined by like-expressing cells to form small clusters (Huang and Moody, 1995, 1997). There is abundant evidence in other developing systems that signals from a cell can induce the fate of neighbors (Dorsky et al., 1997; Hajnal, 1999; Siegfried, 1999; Chitnis, 1999). If amacrine cell clusters were induced to express the same neurotransmitter by a local signal, then clusters should form even in the absence of cell division after the emergence of the first amacrine cell in the cluster. In fact, this was observed for LB-5-HT clusters, implicating cell-cell signaling in forming these clusters. In dramatic contrast, DA and NPY clusters were virtually eliminated when mitoses were blocked during optic vesicle stages, indicating that the cells added to make a cluster are produced by continued cell divisions of a lineage.

The dynamics of cluster formation after repressing cell division at different developmental times provides important insights into the timing of mitoses in amacrine cluster formation. Initial DA amacrine cells were significantly repressed when mitoses were blocked starting at the first morphological indication of the optic vesicle. This suggests that DA cells are born at approximately this time (stages 19-21). Very few clusters were observed when treatment was begun at intermediate optic vesicle stages, whereas $>90 \%$ of embryos contained single DA and NPY cells. In contrast, when treated at the beginning of invagination of the vesicle into a cup, the majority of embryos contained at least one cluster. And, when treated at the definitive cup stages nearly all embryos contained clusters. These data suggest that the majority of the second cells of clusters are born during optic vesicle stages. However the fact that significantly fewer than normal DA and NPY clusters are observed in embryos treated at the optic cup stages indicates that one or more cell divisions occur even later.

\section{Are amacrine-biased eye field progenitors similar to later amacrine and horizontal cell-biased progenitors?}

In agreement with lineage studies performed at later stages, there is no evidence that eye field progenitors are biased to produce only amacrine cells. However, in vitro studies show that some rat optic cup progenitors are biased to produce amacrine and horizontal cells (Alexiades and Cepko, 1997). Other progenitors 
appear biased to produce either rod or bipolar cells (Cepko, 1999; Marrow et al., 1999). The eye field progenitors that produce layered clones, however, may have a different fate choice. The most frequent nonamacrine siblings of amacrine cells were bipolar cells, and in one-half of these clones amacrine and bipolar cells were the only members. The coexpression of amacrine cells with horizontal cells also occurred, but bipolar cells accompanied them in the large majority of cases. The lineal association of amacrine and bipolar cells in this data set does not correspond with the progenitor characteristics described for the later rat retina. But, the preponderance of bipolar cells to the near exclusion of photoreceptors is consistent with the description of progenitors biased to produce either bipolar or rod cells (Marrow et al., 1999). Either the pattern of cell bias is different for eye field versus late retinal progenitors, or layered progenitors produce one bipolar-biased cell and one amacrine and horizontal-biased cell. It will be important to discover the temporal and lineal relationships between the early biased clones of the neural plate described in this report and the later progenitors that have been elucidated in culture paradigms.

\section{REFERENCES}

Adelmann HB (1937) Experimental studies on the development of the eye. IV. The effect of partial and complete excision of the prechordal substrata on the development of the eyes of Ambystoma punctatum. J Exp Zool 75:199-227.

Adler R, Belecky-Adams T (1999) Cell fate determination in the chick embryo retina. In: Cell lineage and fate determination (Moody SA, ed), pp 463-474. New York: Academic.

Alexiades MR, Cepko CL (1997) Subsets of retinal progenitors display temporally regulated and distinct biases in the fates of their progeny. Development 124:1119-1131.

Austin CP, Feldman DE, Ida JA, Cepko CL (1995) Vertebrate retinal ganglion cells are selected from competent progenitors by the action of Notch. Development 121:3637-3650.

Bauer DV, Huang S, Moody SA (1994) The cleavage stage origin of Spemann's Organizer: analysis of the movements of blastomere clones before and after gastrulation in Xenopus. Development 120:1179-1189.

Belliveau MJ, Cepko CL (1999) Extrinsic and intrinsic factors control the genesis of amacrine and cone cells in the rat retina. Development 26:555-566.

Cepko CL (1999) The roles of intrinsic and extrinsic cues and bHLH genes in the determination of retinal cell fates. Curr Opin Neurobiol 9:37-46.

Chitnis AB (1999) Control of neurogenesis-lessons from frogs, fish and flies. Curr Opin Neurobiol 9:18-25.

Dorsky RI, Chang WS, Rapaport DH, Harris WA (1997) Regulation of neuronal diversity in the Xenopus retina by Delta signaling. Nature 385:67-70.

Eagleson GW, Harris WA (1990) Mapping of the presumptive brain regions in the neural plate of Xenopus laevis. J Neurobiol 21:427-440.

Fekete DM, Perez-Miguelsanz J, Ryder EF, Cepko CL (1994) Clonal analysis in the chicken retina reveals tangential dispersion of clonally related cells. Dev Biol 166:666-682.

Hajnal A (1999) Cell fate determination and signal transduction during Caenorhabditis elegans vulval development. In: Cell lineage and fate determination (Moody SA, ed), pp 157-170. New York: Academic.

Harris WA (1997) Cellular diversity in the vertebrate retina. Curr Opin Genet Dev 7:651-658.

Harris WA, Hartenstein V (1991) Neuronal determination without cell division in Xenopus embryos. Neuron 6:499-515.

Hartenstein V (1989) Early neurogenesis in Xenopus: the spatiotemporal pattern of proliferation and cell lineages in the embryonic spinal cord. Neuron 3:399-411.

Holt CE, Bertsch TW, Ellis HM, Harris WA (1988) Cellular determination in the Xenopus retina is independent of lineage and birth date. Neuron 1:15-26.

Huang S, Moody SA (1992) Does lineage determine the dopamine phe- notype in the tadpole hypothalamus: a quantitative analysis. J Neurosci 12:1351-1362.

Huang S, Moody SA (1993) The retinal fate of Xenopus cleavage stage progenitors is dependent upon blastomere position and competence: studies of normal and regulated clones. J Neurosci 13:3193-3210.

Huang S, Moody SA (1995) Asymmetrical blastomere origin and spatial domains of dopamine and neuropeptide $\mathrm{Y}$ amacrine subtypes in Xenopus tadpole retinas. J Comp Neurol 360:2-13.

Huang S, Moody SA (1997) Three types of serotonin-containing amacrine cells in tadpole retina have distinct clonal origins. J Comp Neurol 387:42-52.

Huang S, Moody SA (1998) Dual expression of GABA or serotonin and dopamine in Xenopus amacrine cells is transient and may be regulated by laminar cues. Vis Neurosci 15:969-977.

Jacobson M (1968) Cessation of DNA synthesis in retinal ganglion cells correlated with the time of specification of their central connections. Dev Biol 17:219-232.

Jacobson M, Hirose G (1978) Origin of the retina from both sides of the embryonic brain: a contribution to the problem of crossing at the optic chiasma. Science 202:637-639.

Jacobson M, Hirose G (1981) Clonal organization of the central nervous system of the frog. II. Clones stemming from individual blastomeres of the 32- and 64-cell stages. J Neurosci 1:271-284.

Jensen AM, Raff MC (1997) Continuous observation of multipotential retinal progenitor cells in clonal density culture. Dev Biol 188:267-279.

Klein SL (1987) The first cleavage furrow demarcates the dorsal-ventral axis in Xenopus embryos. Dev Biol 120:299-304.

Lillien L, Cepko CL (1992) Control of proliferation in the retina: temporal changes in responsiveness to FGF and TGF alpha. Development 115:253-266.

MacNeil MA, Masland RH (1998) Extreme diversity among amacrine cells: implications for function. Neuron 20:971-982.

Marrow EM, Belliveau MJ, Cepko CL (1998) Two phases of rod photoreceptor differentiation during rat retinal development. J Neurosci 18:3738-3748.

Marrow EM, Furukawa T, Lee JE, Cepko CL (1999) NeuroD regulates cell fate determination in the developing neural retina. Development 126:23-26.

Masho R (1990) Close correlation between the first cleavage plane and the body axis in early Xenopus embryos. Dev Growth Differ 32:57-64.

Moody SA (1987a) Fates of the blastomeres of the 16-cell Xenopus embryo. Dev Biol 119:560-578.

Moody SA (1987b) Fates of the blastomeres of the 32-cell Xenopus embryo. Dev Biol 122:300-319.

Moody SA (1999a) Cell lineage and fate determination. New York: Academic.

Moody SA (1999b) Testing the cell fate commitment of single blastomeres in Xenopus laevis. In: Advances in molecular biology: a comparative methods approach to the study of oocytes and embryos (Richter J, ed), pp 355-381. New York: Oxford UP.

Moody SA, Bauer DV, Hainski AM, Huang S (1996) Determination of Xenopus cell lineage by maternal factors and cell interactions. Curr Top Dev Biol 32:103-138.

Moore KB, Moody SA (1999) Animal-vegetal asymmetries influence the earliest steps in retinal fate commitment in Xenopus. Dev Biol 212:25-41.

Newport J, Kirschner M (1982) A major developmental transition in early Xenopus embryos. II. Control of the onset of transcription. Cell 30:687-696.

Nieuwkoop PD, Faber J (1994) Normal table of Xenopus (Daudin). New York: Garland.

Perron M, Harris WA (1999) Cellular determination in amphibian retina. In: Cell lineage and fate determination (Moody SA, ed), pp 353-368. New York: Academic.

Reese BE, Tan SS (1998) Clonal boundary analysis in the developing retina using $\mathrm{X}$-inactivation transgenic mosaic mice. Semin Cell Dev Biol 9:285-292.

Reh TA (1992) Cellular interactions determine neuronal phenotypes in rodent retinal cultures. J Neurobiol 23:1067-1083.

Reh TA, Levine EM (1998) Multipotential stem cells and progenitors in the vertebrate retina. J Neurobiol 36:206-220.

Reh TA, Tully T (1986) Regulation of tyrosine hydroxylase-containing amacrine cell number in the larval frog retina. Dev Biol 114:463-469. 
Saha MS, Grainger RM (1992) A labile period in the determination of the anterior-posterior axis during early neural development in Xenopus. Neuron 8:1003-1014.

Siegfried E (1999) Role of Drosophila wingless signaling in cell fate determination. In: Cell lineage and fate determination (Moody SA, ed), pp 249-271. New York: Academic.

Spemann H (1938) Embryonic development and induction. New Haven, $\mathrm{CN}$ : Yale UP.

Turner DL, Snyder EY, Cepko CL (1990) Lineage-independent determination of cell type in the embryonic mouse retina. Neuron 4:833-845.

Waid DK, McLoon SC (1995) Immediate differentiation of ganglion cells following mitosis in the developing retina. Neuron 14:117-124.

Waid DK, McLoon SC (1998) Ganglion cells influence the fate of dividing retinal cells in culture. Development 125:1059-1066.

Watanabe T, Raff MC (1990) Rod photoreceptor development in vitro: intrinsic properties of proliferating neuroepithelial cells change as development proceeds in the rat retina. Neuron 2:461-467.

Wetts R, Fraser SE (1988) Multipotent precursors can give rise to all major cell types of the frog retina. Science 239:1142-1145.

Wetts R, Fraser SE (1989) Slow intermixing of cells during Xenopus embryogenesis contributes to the consistency of the blastomere fate map. Development 105:9-15.

Wetts R, Serbedzija GN, Fraser SE (1989) Cell lineage analysis reveals multipotent precursors in the ciliary margin of the frog retina. Dev Biol 136:254-263.

Williams RW, Goldowitz D (1992a) Structure of clonal and polyclonal cell arrays in chimeric mouse retina. Proc Natl Acad Sci USA 89:1184-1188.

Williams RW, Goldowitz D (1992b) Lineage versus environment in embryonic retina: a revisionist perspective. Trends Neurosci 15:368-373. 